\title{
POLÍTICA AGRARIA Y POSCONFLICTO EN COLOMBIA' \\ (Agricultural policy and post-conflict in Colombia)
}

Dustin Tahisin Gómez Rodríguez dgomez@usbbog.edu.co

Ehyder Mario Barbosa Pérez ${ }^{3}$ ebarbosa@igac.gov.co

William Eduardo Rojas Velasquez ${ }^{4}$ william_rojasvel@cun.edu.co

Fecha de recepción: 16 julio de 2015 Fecha de aceptación: 5 octubre 2015

El objetivo principal del presente artículo es analizar la literatura que se ha desarrollado en los diferentes sectores sociales sobre la política agraria desde la promulgación de la Ley 1448 de 201 I como factor relevante del posconflicto. Para ello, se utilizaron las bases de datos avaladas por Colciencias como se indagó diferentes documentos publicados por instituciones privadas como públicas. La metodología a utilizar es de corte cualitativo, analítico descriptivo. La principal conclusión es que los actores sociales han generado tanto espacios de disertación como de exposición sobre la problemática multidimensional del agro colombiano y cómo estas dimensiones deben ser concertadas para generar un paz duradera, donde el diálogo con las diferentes comunidades étnicas, políticas y económicas sean baluartes para reconstruir un tejido social resquebrajado por décadas de injustica e inequidad.

Palabras clave: ley 1448 de 20II, política agraria, posconflicto, víctimas.

Abstract

The main objective of this article is to analyze the literature that has been developed in different social sectors about agricultural policy since the promulgation of the Law 1448 of 201 I as a relevant factor in the post-conflict. To do this, databases supported by Colciencias are used also different documents published by private and public institutions are required. The methodology used is qualitative, descriptive analytical. The main conclusion is that social actors have generated spaces of dissertation as exposure of the multidimensional problematic of Colombian agriculture and how these dimensions must be specified to generate a durable peace, where dialogue with the different ethnic, political, and economic communities are bulwarks to rebuild a social fabric shattered by decades of injustice and inequality.

Keywords: law 1448 of 20II, agrarian policy, post-conflict, victims.

'Proyecto de investigación Desarrollo, territorio y postconflicto, realizado en colaboración entre la Universidad San Buenaventura, sede Bogotá, el IGAC, y la de la Corporación Unificada de Educación Superior,CUN.

${ }^{2} E$ conomista de la Universidad de la Salle, Especialista en Psicología Educativa de la Universidad Católica de Colombia, Magíster en Estudio y Gestión del Desarrollo, Universidad de la Salle. Docente asociado de la Facultad de Ciencias Jurídicas, Políticas y Económicas de la Universidad San Buenaventura, líder de investigación de la Escuela de Ciencias Administrativas de la CUN.

${ }^{3}$ Economista de la Universidad de la Salle. Magister en Estudio y Gestión Del Desarrollo de la Universidad de La Salle. Asesor en temas de contratación pública y Hacienda Pública de la Dirección General del IGAC (Instituto Geográfico Agustín Codazzi), Bogotá, Colombia.

${ }^{4}$ Filósofo de la Universidad Nacional de Colombia. Estudiante de la Maestría en Filosofía Política de la Universidad Nacional de Colombia. Docente investigador del programa de Negocios Internacionales, CUN. 


\section{INTRODUCCIÓN}

a concatenación entre el discurso económico y L la política agraria en Colombia ha generado transformaciones sociales que permean tanto a las instituciones como a los actores que participan en ella; desde esa perspectiva, las investigaciones que toman como línea argumentativa el agro se han focalizado en enaltecer el discurso desde cada uno de sus componentes y de sintonizarla con los requerimientos de un mundo que tiende a ser globalizado (De Roux, 2012; Chiriboga, 1991; Kalmanovitz y López, 2006, y (García, 1967 y 1967b). En consecuencia, el presente Gobierno ha concebido que una forma en la cual el desarrollo y el crecimiento económico del país puedan cumplir con uno de sus objetivos, que es el de mejorar la calidad de vida de los colombianos, es a través de los diálogos que se están efectuando en La Habana, donde una variable que puede contribuir a la paz es el desarrollo de la Ley 1448 de 201I, la cual, además de resarcir a las víctimas del conflicto, es también un procedimiento que rige la restitución de tierras, esta última como factor económico y político de la distribución de la propiedad, que ha contribuido a la desigualdad de una gran proporción de la sociedad colombiana, dadas las grandes injusticas a las que se ha visto presionada la población rural gracias a los actores, tanto ilegales como legales, que la usurpan (Cepeda y Uribe, 2014; Restrepo y Bernal, 2014, y Bernal, 1999); de ahí que:

La sociedad colombiana enfrenta una compleja pero inaplazable tarea: la implementación de un programa de restitución de las tierras y bienes que han sido expropiados a través de la intimidación y la violencia. Sin lugar a dudas, los conflictos derivados de la concentración de la propiedad de la tierra en Colombia constituyen uno de los factores estructurales más importantes para explicar el origen y la persistencia de la violencia política. Además, la reparación de las víctimas del despojo y el abandono no es obligación moral sino, además, un deber jurídico ineludible importante (Sánchez y Uprimmy, 2010, p. 193).

En efecto, al desagraviar a las víctimas como al empoderar las leyes, dadas sus dispersiones, se abren caminos analíticos frente a lo acontecido con la Ley 160 de 1994, y, del mismo modo, se clarifica la función social y ecológica de la propiedad, como uno de los símbolos más importantes sobre los cuales se funda una buena parte el futuro de la reforma agraria en el posconflicto.
En virtud de identificar cómo sintetizar el problema agrario, se generan dudas y críticas a los procedimientos y ejecuciones a través de los que se ha venido gestionando, gracias a la gran cantidad de necesidades de las que adolece el país en materia del incremento de su área cultivable, como de la autosuficiencia alimentaria, sin que se desconozca el desarrollo de una agroindustria altamente competitiva (FAO, 2012, y Restrepo y Bernal, 2014). Por ello, administrar eficientemente los recursos implica entender cómo dinamizar los procesos geopolíticos en los cuales se fecunda la dotación de tierra en Colombia, sin olvidar, como lo acotó la premio Nobel de Economía, Elinor Ostrom, en su libro El gobierno de los bienes comunes, que: "Ni el Estado ni el mercado han logrado con éxito que los individuos mantengan un uso productivo, de largo plazo, de los sistemas de los recursos naturales" (2000, p. 26).

Por consiguiente, el objetivo del presente artículo es analizar la literatura que se ha desarrollado, tanto en los diferentes entes académicos como en el sector real, sobre la pertinencia de la política agraria desde la promulgación de la Ley 1448 de 201 I como factor relevante para el posconflicto. Para ello, se utilizaron bases de datos avaladas por Colciencias y se consultaron diferentes documentos publicados por instituciones privadas y públicas, a través de una metodología de corte cualitativo, analítico-descriptivo. El artículo se divide en la presente introducción, seguida de la identificación de la Ley 1448 de 201I, luego una interpretación de la categoría posconflicto, a continuación un análisis sistemático de la literatura examinada, para terminar con unas breves conclusiones.

\section{Metodología}

La metodología utilizada es de corte cualitativo, analítico-descriptivo. Se realizó una revisión de literatura especializada, sobre las bases de datos avaladas por Colciencias como Ebsco, Redalyc, ProQuest, etc. De ahí que esta clase de enfoques se aleja de la experimentación, del control, para ir en sintonía con la concepción de la interpretación como instrumento del investigador cualitativo, en virtud de que "(...) en última instancia el investigador termina por dar su visión personal" (Stake, 1998, p. 46).

\section{LEY |448 DE 201/5}

El conflicto interno armado en Colombia lleva aproximadamente sesenta y cuatro años, de lo cual 
se sigue que ha contribuido, entre otros aspectos, al bajo crecimiento económico y a niveles inaceptables de desarrollo, dadas las potencialidades de los recursos humanos como de las materias primas que posee el país (Cepeda y Uribe 2014). No se debe olvidar, además, que ha expuesto al Estado colombiano a una continua violación de los derechos humanos; en efecto, el actual Gobierno, en su primer periodo, inició un proyecto legislativo que tiene como finalidad la reparación administrativa de todos los individuos que hayan sido víctimas del conflicto armado (PNUD, 2011) y (Universidad de San Buenaventura, 2014). En consecuencia, sancionó la Ley 1448 de 201I, denominada Ley de Víctimas y Restitución de Tierras, con injerencia asidua del Ministro de Agricultura de ese entonces, Juan Camilo Restrepo, y su gabinete. La Ley 1448 busca garantizar a las víctimas del conflicto interno armado "la justicia, la verdad y la reparación integral de sus posesiones", sin olvidar la "no repetición" del hecho de injustica e inequidad que incumba en los suelos colombianos (Chávez y Vargas, 2014).

De igual manera, la Ley 1448 de 201 I aspira a forjar diálogos y espacios de reconciliación, así como a resarcir a las víctimas por medio de una reparación integral, desde lo económico, que fomente la educación, la salud, brindando asistencia humanitaria y un acompañamiento continuo en el proceso. Con la "justicia transicional" el Gobierno busca, entre otras cosas, cambiar la mirada que se tiene en el exterior frente a la vulneración de los derechos humanos en el país; por ello, el Estado colombiano ha venido organizando mecanismos administrativos que favorezcan la ejecución de políticas públicas, una de cuyas tipologías es que la Ley sea progresista y ágil, y que no victimice de nuevo a las víctimas (Universidad de San Buenaventura, 2014) y (Pinzón, 2012).

El acceso a la Ley de Víctimas y Restitución de Tierras posee unas características que hacen de ella la primera en su clase. Entre sus principales objetivos está el de reconocer que las víctimas son personas que, individual o colectivamente, sufrieron daño de manera directa con hechos que tuvieron relación con el conflicto armado, siempre que los mimos se hayan producido a partir de 1985. Este beneficio, si se puede utilizar el término, también es extensivo a los familiares que presenten las peculiaridades antes expuestas; lo que quiere decir, por ejemplo, que los hijos nacidos como consecuencia de una violación sexual tienen derechos como víctimas. De igual manera, la Ley presenta un avance significativo en el reconocimiento diferencial de género, dado que la población femenina fue también invisibilizada y discriminada (Rodríguez, 2014). De igual forma, la reparación tiene cinco ejes o componentes integradores: la rehabilitación, la indemnización, la satisfacción, la garantía de no repetición y la restauración de los bienes por parte del victimario (PNUD, 20ll).

\section{POSCONFLICTO}

El concepto posconflicto se puede definir como el periodo que sigue cuando se avanza o se supera, en determinados momentos o totalmente, el cese de los conflictos armados en un Estado. El término puede entenderse como la reducción del número de muertes relacionadas con el conflicto desde la perspectiva de un umbral determinado, lo cual se identifica con el estatus de que el conflicto no está activo (Ugarriza, Nuzzio, Orozco, Rodríguez, Martínez y Gutiérrez, 2013). No obstante, no es solo la cesación del conflicto, y por ende la reducción de muertes, sino que es una reconfiguración del tejido social a través de fenómenos como la desmovilización, la reintegración, la reconciliación, la prevención de la violencia, la estabilización económica, la participación de los diferentes sectores, la justicia transicional, la reparación de las víctimas, la solidificación y el fortalecimiento de la paz con la comunicad civil, en un mundo que tiende a ser cada vez más globalizado (Rettberg, 2003 y 2012; Cetil, 2009, y Boutros-Ghali, 1992). En general, es la construcción de una nueva sociedad, en la que sus actores tienen voz y voto (Varona, 2014). Precisamente, el proceso de paz que se está gestionando en La Habana por parte del Gobierno colombiano va en concordancia con los derechos humanos y el Derecho Internacional Humanitario, en virtud de que son normas que ayudan a resarcir los derechos de las víctimas del conflicto armado. En consecuencia, el Estado como garante debe ser consecuente con sus estrategias de diálogo (Combita , Delgadillo y Torres, 2013, p. 33).

\section{DOCUMENTOS QUE PROVIENEN DE}

\section{UNIVERSIDADES COLOMBIANAS}

La academia colombiana es uno de los actores que también puede aportar en la consecución de la paz por medio de la investigación, la capacitación del capital humano para el posconflicto, desde el punto de vista de las necesidades del aparato productivo, así como de la construcción del entramado social a través de la crítica y el debate de la cultura. Entre los documentos que se analizaron se identificaron el del "III Foro Colombiano en Construcción de la paz", que se llevó a cabo el 14 de marzo de 2013 en la Universidad Jorge Tadeo Lozano, el cual tuvo como panelistas a Ricardo Sabogal, de la Unidad para la Restitución de Tierras; Absalón 
Machado, de la Universidad Nacional de Colombia; Alejandro Éder, de la Agencia Colombiana para la Reintegración, y Antonio Navarro Wolf congresista del Partido Verde, quienes disertaron sobre la alternativa que representa el posconflicto. Entre sus reflexiones, quedó clara la problemática de la restitución de tierras, pues proviene de antes de los orígenes de las FARC; además se evidenció que el Estado colombiano tiene una deuda pendiente con las víctimas y que para que la paz sea duradera es necesario que sea sostenible. Por consiguiente, una forma de ampliar el debate es comprender quiénes son las víctimas y quiénes los victimarios. En efecto, la construcción de la paz es una edificación de diferentes actores en la que el Estado también tiene su parte de gestión, por ejemplo, a través de su presencia en el territorio, no solamente con la Policía y el Ejército, sino también con educación y salud, que son características de un Estado de derecho (Sabogal, Machado, Éder, y Navarro, 20 I3). Igualmente, Gutiérrez (2014) enfatiza que los costos de transacción en los que debe incurrir el Estado colombiano desde el punto de vista de la restitución de tierras son muy onerosos, pues en el país jamás ha existido una profunda reforma agraria.

Sin embargo, adelantar este proceso es generar un acervo para contribuir a una paz perdurable. De modo similar, Estrad y Rodríguez (2014) realizaron un estudio desde el punto de vista político y jurídico sobre la restitución de tierras de la Ley 1448, en el cual concluyen que la Ley de Víctimas y Restitución de Tierrasha evolucionado de la idea original de protección a restitución. Para llegar a esta conclusión desarrollaron tres distintivos: (a) reconstruyen latrayectoria del proceso, (b) evidencian los avances técnicos que posibilitan dicha Ley, y (c) analizan las lógicas estatales que dirigen el proceso. En el mismo sentido (Serrano y Acevedo, 2013) señalan lo ecuánime de la Ley, en virtud de que la norma agraria facilita el proceso de restitución, así como la delimitación de las zonas denominadas baldíos, a través de la oficialización de matrículas inmobiliarias y de la actualización del catastro; los autores agregan que "las políticas de restitución han puesto de presente situaciones de corrupción administrativa no denunciadas anteriormente, relacionados con la titulación de baldíos, lo cual es beneficioso para depurar el camino de acceso a la propiedad campesina en Colombia" (p. 564).

En el mismo sentido, la investigación que realizó la Universidad de Caldas, De campesinos a empresarios: la retórica neoliberal de la política agraria en Colombia, indica que se ha vigorizado la producción empresarial de cultivos tropicales; sin embargo, la economía campesina se ha reducido drásticamente, lo que ha contribuido a la pauperización de la calidad de vida del campesino promedio (Tobasura, 20ll). De lo anterior se sigue que procesos como este podrían no ayudar a cristalizar un posconflicto $y$, por ende, ser un obstáculo para la Ley de Víctimas y Restitución de Tierras,gracias a que la precarización de los ingresos de los campesinos impacta negativamente en el agro como fuente de trabajo. En contraste, el documento de Franco y De los Ríos (20II) explica que las reformas agrarias han tenido once periodos y cómo estas han evolucionado desde ser un enfoque simple, en el que solo se abordaba la propiedad de la tierra, a ser un multiplicador de herramientas en el que se identifica un enfoque integral; es decir, que hay correspondencia entre los procesos agrarios, lo económico y lo político por medio de la planificación de las necesidades básicas del campesinado, lo que permiten la participación de la banca, y el acompañamiento técnico y empresarial (Cinep, 2013).

Con el mismo propósito, Tadloui (2012), desde su pregunta-problema "iPor qué una tierra con alta potencialidad productiva entregada a sujetos de la reforma agraria llegó a generar condiciones desfavorables para sus beneficiarios?" (p. II5), realiza un estudio de caso de restitución de las tierras de Sandrana y Samaría, en el cual se ejecuta un marco que, según los autores, es imperativo para que se equipare el factor tierra como una variable de desarrollo para estas comunidades, señalando que la organización y la construcción comunitaria son también características para el desarrollo de proyectos productivos que, a su vez, son refuerzos para el posconflicto. Por otra parte, el estudio de Cardozo (20II) enunció cómo una serie de poderes ajenos al agro colombiano han ejercido violencia física y social en el desarrollo del mismo, lo cual ha contribuido a la ineficiencia y a acrecentar tanto la injusticia como la inequidad. El autor también indica cómo los mismos actores rurales han venido gestionando procesos en pro de su localidad y cómo esto, a su vez, contribuye a mejorar su calidad de vida. Por último, Infante (2014) resalta la importancia de la educación como estrategia a corto y largo plazo en la reconstrucción del tejido social, en virtud de que provee protección psicosocial, cognitiva y física que coadyuvan al desarrollo social y económico del país, señalando que la Ley dimensiona este enfoque como propuesta de cualificación de las víctimas que retornan a sus tierras.

\section{DOCUMENTOS QUE PROVIENEN DE DISTINTOS}

\section{SECTORES NO UNIVERSITARIOS}

En virtud de las negociaciones que se están efectuando en La Habana por parte del Gobierno 
actual, varios sectores han contribuido con ponencias, conversatorios y foros para construir la paz anhelada. Entre los más recientes, se han podido identificar el ciclo de conversatorios, auspiciados por Planeta y $\mathrm{Paz}^{6}$ y $\mathrm{PCS}^{7}$, realizados en 2012 y que tuvieron como invitados a Carlos Ancizar Rico, Eduardo Arias y Teófilo Acuña, entre otros, quienes con sus aportes contribuyeron en el debate a la crítica del proceso agrario en Colombia, así como a las posibilidades del posconflicto. Entre sus conclusiones se destaca la necesidad de que las propuestas que nacen de los diferentes actores que se empoderan desde las distintas regiones sean valoradas por el Gobierno, en virtud de que al construir el tejido social es imperativo salirse de los planteamiento institucionales y focalizarse más en construir redes con organizaciones afrocolombianas, indígenas y campesinas, debido a que la disputa de la tierra en Colombia tiene enfoques multidimensionales. Esto, sin desconocer la relación del modelo económico con la inequidad e injusticia imperante en el campo, donde los Gobiernos han concebido el agro solo como objeto de la explotación de los recursos de corte extractiva, como la agroindustria, lo que oblitera las formas de entender divergentes de las comunidades afectadas, las cuales se constituyen como variables negativas para el entramado social en el posconflicto si no se dimensionan estas cosmovisiones (Planeta y Paz, y PCS, 2012, pp. 123|3I).

De igual forma, el Incoder, con el apoyo de la Corporación Latinoamericana "Misión Rural", forjó un documento como estrategia de comunicación de la complejidad de la propiedad de la tierra en Colombia. A través de una serie de entrevistas con personas como Luis Jorge Garay, Absalón Machado y Miguel Antonio Calambás, entre otros, de testimonios de campesinos y líderes comunales, así como de los artículos de Cecilia López, Lorena Hoyos y Alfredo Molano, evidenciaron, desde la coyuntura actual, la necesidad de generar soluciones a esta problemática que tiene décadas en la sociedad colombiana. En el mismo sentido, se puede identificar, a lo largo del escrito, que el proceso de la paz que se tramita entre el Gobierno y las FARC sitúa en su agenda la necesidad de reflexionar sobre el entramado social colombiano; sobre cómo la tierra es detonante de inequidad e injusticia social y cómo la Restitución de Tierras, según se ha expuesto, es un baluarte que beneficia y socorre a los pobladores del campo del olvido social, orientado hacia las minorías étnicas y las comunidades campesinas usurpadas por elementos armados por fuera de la Ley (Incoder, 2013).
Por otra parte, el Banco de la República efectuó una investigación sobre la relación entre las aspiraciones económicas de los colombianos y el conflicto armado. A través de modelos matemáticos concluye que las personas que viven cerca del conflicto armado, o conviven con él, tienden a tener menores aspiraciones económicas que los que no, sin olvidar que existen variables que también repercuten en estas aspiraciones como las edades, el entorno de desigualdad entre sus contemporáneos y la inversión estatal en sus municipios (Ayala, 2014), lo cual reafirma la importancia tanto del empoderamiento del Estado en estos lugares como de la propiedad de las tierras usurpadas, como contribución a una paz duradera en el posconflicto. Por otro lado, existen otras particularidades del posconflicto como la desmovilización de los insurgentes y la posibilidad de que algunos de ellos retomen sus tierras cuando regresen a sus lugares de origen. Desde esa perspectiva, en la revista Criminalidad, de la Policía Nacional, específicamente de la Dijín, la Asesora del Centro de Investigaciones Criminológicas de la Dirección Central de Policía Judicial realizó un pequeño estudio sobre esta población, comparándola con otros países como Camboya y el Salvador. En su investigación, la autora sintetiza que es necesaria la capacitación de estos individuos para que muchos de ellos no regresen a bandas delincuenciales; del mismo modo, resalta la importancia de la sostenibilidad del posconflicto, es decir la sustentabilidad, dado los recursos escasos de la nación y lo imperioso de crear sinergias con entes multilaterales como la OEA y la ONU para canalizar fondos. Asimismo, afirma que hay que realizar estrategias a través de las cuales se empodere a estas personas, una de las cuales puede ser la entrega de armas con beneficio, es decir, la recompra o programas de intercambio (Bello, 2012).

El informe del Ministerio de Agricultura y Desarrollo Rural de 2013 señala que "los conflictos sobre la tierra han sido la causa de la guerra en Colombia" (p. 3); que superar el conflicto debe pasar por "reparar a las víctimas" para "lograr el desarrollo rural", y que, a su vez, "modernizar este país requiere inevitablemente que se aborden las diversas problemáticas de las tierras y los territorios y se regularicen los vicios del pasado" (p. 25). De lo cual se sigue que "es urgente el reconocimiento de los derechos a la tierra de los campesinos, desplazados, indígenas y afrodescendientes" (p. 25). Explicando que "formalizar los derechos sobre la tierra a favor de esta población, garantizará el mejoramiento de su condición de vulnerabilidad" (Restrepo, 2013, p. 25). En 
otras palabras, el fortalecimiento de una sociedad más equitativa y justa se basa en mejorar y resarcir la calidad de vida de los colombianos del agro, lo que favorecería el desarrollo y el crecimiento económico del país (Galtung, 1975).

De la misma forma, la Ley de Víctimas y Restitución de Tierrases un factor que puede dinamizar la economía rural (Reyes, 1978), dada la filtración, sobre todo desde la década de los ochenta, de grupos de derecha y de izquierda, precisando que el rezago institucional en estos territoritos influye negativamente en la informalidad de los derechos de propiedad, o potencializa, ante lo cual, argumentan los autores, se puede mejorar, siempre y cuando esté acompañada y visualizada desde un enfoque multidimensional, en virtud de la gran cantidad de aristas que tiene la posesión de tierras por parte de sus verdaderos dueños (Balcázar y Rodríguez, 2013). Para terminar, conciben que el desarrollo agrícola debe inscribirse en un marco normativo, el cual, desde una nueva visión del desarrollo rural, debe propender por reducir las desigualdades sociales (Tadloui, 20I2), siendo una de sus metas la mayor equidad y la distribución de los ingresos, acotando la importancia de dinamizar el campo por medio de la cualificación del capital humano desde lo laboral en prácticas ambientales, que son otro detonante para que se dé la paz (Becerra y Hernández, 2013). En el mismo sentido, Restrepo (2011, p. 18) subraya que el Gobierno del presidente Santos (20102014) ha ido agenciando una política de tierras que no sea antagónica con el desarrollo de la agricultura en aras de la exportación; sin embargo, para llegar a ello, reitera la necesidad de la trazabilidad de la Ley de Víctimas y Restitución de Tierras, en virtud de la deuda gigantesca que tiene el país con más de trescientos cincuenta mil familias a las cuales, según lo indican los datos, les arrebataron más de dos millones de hectáreas, señalando, como lo expuso el Director General del Instituto Geográfico Agustín Codazzi, Juan Antonio Nieto Escalante, que "el posconflicto ya inició".

Tenemos a puertas la suscripción de un convenio de paz que va a beneficiar a todos los colombianos, y el cual ha sido ratificado en las urnas a través de la reelección. Con esto, todo el país empezará una gran transformación, que tiene que ver con el ordenamiento territorial. En este tema, el IGAC es ficha clave, ya que cuenta con los insumos básicos para que sobre ellos se construyan las capas de información y se tomen las medidas y las decisiones más acertadas" (IGAC, 2014, p. I).

Lo anterior muestra cómo esta entidad está haciendo los esfuerzos pertinentes para generar un catastro rural a la vanguardia, en consonancia con de la Ley de Víctimas y Restitución de Tierras.
En síntesis, las negociaciones que se están gestando en La Habana necesitan de acuerdos generales tanto sobre el régimen transicional como desde lo político, con el fin de resarcir a las víctimas del conflicto armado:

Esto requiere la persecución penal de los máximos responsables de ambas partes por los peores crímenes; una comisión de la verdad fuerte e independiente; la reparación integral a todas las víctimas que incluya más que una compensación financiera; y medidas para garantizar la no repetición. Las posibilidades de que esto se consiga están mejorando gradualmente. Como los diálogos han cobrado fuerza, y la idea de que el conflicto podría llegar a un final negociado se ha convertido en una proposición realista, ambas partes han avanzado en reconocer su responsabilidad por los crímenes cometidos durante el conflicto (ICG, 20I3, p. 57).

\section{CONCLUSIONES}

La academia debe aportar investigaciones que dinamicen el escenario del posconflicto como una posibilidad para cerrar la brecha existente entre lo rural y lo urbano, para que la política agraria y el discurso económico estén alineados con mejores prácticas sustentables de justicia y de paz. En el mismo sentido, las instituciones educativas deben generar sinergias con el aparato productivo y los diferentes sectores de la sociedad colombiana para contribuir en la construcción de una sociedad más equitativa y justa, elaborando contribuciones que dinamicen y potencialicen el recurso humano que se va a desmovilizar.

La Ley de Víctimas y Restitución de Tierrases un instrumento multidimensional, único en su clase, que contribuye a mejorar y resarcir a las víctimas del conflicto armado en Colombia. Entre sus propósitos está ayudar a zanjar las injusticias y reparar las vejaciones que sufrieron estas personas por parte de elementos armados por fuera de la ley o de los grandes terratenientes en busca de dinamizar sus acrecentadas fortunas.

El Estado colombiano se encuentra en un proceso de transformación de cada uno de los aspectos de la vida del ciudadano. Si finalizan de manera exitosa los diálogos de La Habana, tendríamos una buena oportunidad para mejorar las condiciones de vida de la sociedad en su conjunto. En consecuencia, cada uno de los actores sociales debería contribuir apoyando esta oportunidad para acabar con uno de los grandes flagelos en la historia reciente del país como lo es el conflicto armado interno \& 


\section{Referencias bibliográficas}

Ayala, J. (2014). Aspiraciones económicas, conflicto y trampas de pobreza en Colombia. (Documentos de trabajo sobre economía regional, No. 212). Cartagena: Banco de la República - CEER. Versión en línea disponible en http://www.banrep.gov.co/sites/default/files/publicaciones/archivos/dtser_212.pdf

Balcázar, A. y Rodríguez, C. (2013). Tierra para uso agrario. En J. J. Perfetti (coord.) Políticas para el desarrollo de la agricultura en Colombia (pp. 65-I I4). Bogotá, D. C.: SAC y Fedesarrollo. Versión en línea disponible en http://www.fedesarrollo.org.co/wp-content/ uploads/2013/07/Libro-SAC_Web.pdf

Bernal, F. (1999). Poder y crisis institucional del campo. Bogotá, D. C.: Misión Rural.

Bello, C. (2012). Posconflicto y desmovilización: Comparativo de las experiencias en Colombia, Camboya y el Salvador. Revista Criminalidad: Policía Nacional de Colombia, Dijín, 48(I I), 232-24I. Versión en línea disponible en http://www.policia.gov.co/imagenes_ponal/dijin/ revista_criminalidad/vol48/II.pdf

Boutros-Ghali, B. (1992). An agenda for peace.Nueva York: ONU.

Cardozo, O. (Julio-diciembre de 20II). Incidencia de poderes exógenos en las políticas públicas y en el sector rural en Colombia. Revista Apuntes del CENES, 30(52), 103-106.

Centro de Investigación y Educación Popular -Cinep. (2013). Aportes a los diálogos de paz: Participación política y negociaciones de paz (Documento 3). Bogotá, D. C.: Cinep - Programa por la Paz. Versión en línea disponible en http://www.jesuitas.org.co/documentos/74.pdf Cepeda, I. y Uribe, A. (20I4). Por las sendas del uberrismo. Bogotá, D. C.: Grupo Zeta.

Chávez, B. y Vargas, B. (Julio-diciembre de 2014). Significado de restitución de tierras en población desplazada en Soacha. Tendencias \& Retos, I9(2), I23-136.

Chetail, V. (2009).Post-conflict peace building: A lexicon. Nueva York: Oxford University Press.

Chiriboga, M. (1991). Hacia una modernización democrática e incluyente de la agricultura de América Latina y El Caribe. En A. Machado (comp.), Seminario Internacional: Desarrollo rural y apertura económica (pp. 14-72). Bogotá, D. C.: Fondo DRI e IICA. Versión en línea disponible en http://repiica.iica.int/DOCS/B I I 29E/B I I 29E.PDF

Combita, L., Delgadillo, L. y Torres, S. (2013). El posconflicto en Colombia: Una mirada hacia los diferentes procesos de paz (tesis de grado). Facultad de Ciencias Empresariales, Corporación Universitaria Minuto de Dios. Bogotá, D. C., Colombia. Versión en línea disponible en http://www.corteidh.or.cr/tablas/usuario/sierra/Posconflicto/El\%20posconflicto\%20en\%20Colombia.pdf

De Roux, F. (2012). Palabras finales del foro "Desarrollo IntegralAgrario Regional", Bogotá diciembre de 2012 [documento en línea]. Recuperado de http://pnud.org.co/img_upload/6I62646I626434343535373737353535/20I2/DISCURSO\%20-\%20PADRE\%20FRANCISCO\%20DE\%20ROUX\%20EN\%20FORO\%20DESARROLLO\%20INTEGRAL\%20AGRARIO\%20REGIONAL.pdf

Estrad, M. del R. y Rodríguez, N. M. (2014). La política de tierras para la población desplazada 200I-20I I: De la protección a la restitución. Estudios Socio-Jurídicos, I6(I) 75-I 19. Versión en línea disponible en http://revistas.urosario.edu.co/index.php/sociojuridicos/article/view/ esj16.I.2014.02/2378

Franco, A. y De los Ríos, I. (20I I). Reforma Agraria en Colombia: Evolución histórica del concepto. Hacia un enfoque integral actual. Cuadernos de Desarrollo Rural, 8(67), I2-30. Versión en línea disponible en http://www.scielo.org.co/pdf/cudr/v8n67/v8n67a05.pdf

García, A. (1967a). Dinámica de las reformas agrarias en América Latina. Santiago de Chile: Icira.

García, A. (1967b). Reforma agraria y economía empresarial en América Latina. Santiago de Chile: Universitaria.

Galtung, J. (1975). Three approaches to peace: Peacekeeping, peacemaking and peace building. En Peace, war and defence: Essays in peace research (pp. 282-304). Copenhage: Christian Heljers.

Gutiérrez, J. A. (2014). Costos sociales de transacción de la Ley de Restitución de Tierras en Colombia: Un país sin reforma agraria. Revista Jurídicas CUC,IO(I),I57-I96. Versión en línea disponible en http://revistascientificas.cuc.edu.co/index.php/juridicascuc/article/view/464/ pdf_48

Hernández, A. y Becerra, A. (20I3). Capital básico para la agricultura colombiana. En J. J. Perfetti (coord.), Políticas para el desarrollo de la agricultura en Colombia (1 17-185). Bogotá, D. C.: SAC y Fedesarrollo. Versión en línea disponible en http://www.fedesarrollo.org.co/ wp-content/uploads/2013/07/Libro-SAC_Web.pdf 
Infante, A. (2014). El papel de la educación en situaciones de posconflicto: Estrategias y recomendaciones. Hallazgos: Revista de Investigaciones, Año II (2I), 223-246. Versión en línea disponible http://revistas.usantotomas.edu.co/index.php/hallazgos/article/view/I I56/I390 Instituto Colombiano de desarrollo Rural -Incoder y Corporación latinoamericana "Misión Rural” (2013). Pensar la Tierra. Bogotá, D. C.: Carvajal. Versión en línea disponible en http://www.misionrural.net/misionimg/Libro\%20Pensar\%20la\%20Tierra.pdf

Instituto Geográfico Agustín Codazzi -IGAC. (20I4). IGAC será una entidad clave en el postconflicto de Colombia: Juan Antonio Nieto Escalante [documento en línea]. Recuperado de http://www.igac.gov.co/wps/wcm/connect/ad8b080044d3b65293c59373ea9aa578/IGAC + ser\%C3\%AI + una+entidad+clave+en+el+postconflicto+de+Colombia.pdf?MOD=AJPERES

International Crisis Group -ICG (2013). Justicia transicional y los diálogos de paz en Colombia: Informe sobre América latina No. 49 [documento en línea]. Recuperado de http://www.crisisgroup.org/ /media/Files/latin-america/colombia/049-transitional-justice-and-colombiaspeace-talks-spanish.pdf

Kalmanovitz, S. y López, E. (2006). La agricultura colombiana en el siglo XX. Bogotá, D. C.: Banco de la República.

Ley 1448 de 20I I-Por la cual se dictan medidas de atención, asistencia y reparación integral a las víctimas del conflicto armado interno y se dictan otras disposiciones. Diario Oficial (No. 48.096 de 10 de junio de 20I I) [documento en línea]. Recuperado de http://www.secretariasenado.gov.co/senado/basedoc/ley_l448_20II.html

Organización de la Naciones Unidas para la Alimentación y la Agricultura -FAO. (2012). Directrices voluntarias sobre la gobernanza responsable de la tenencia de la tierra, la pesca, los bosques en el contexto de la seguridad alimentaria.Roma: CFS -FAO. Versión en línea disponible en http://www.fao.org/docrep/0I6/i280Is/i280 Is.pdf

Ostrom, E. (2000). El gobierno de los bienes comunes: La evolución de las instituciones de acción colectiva. México, D. F.: Fondo de Cultura Económica.

Palacios, M. (20II). ¿De quién es la tierra?: Propiedad, politización y protesta campesina en la década de 1930. Bogotá, D. C.: Universidad de los Andes.

Pinzón, J. C. (Febrero de 2012). La restitución de tierras es una cruzada contra la ilegalidad. PNC: Periódico de la Policía Nacional de Colombia, (9) p. 3 [documento en línea]. Recuperado de http://www.policia.gov.co/home_html5/pdf/ediciones-pnc/pnc9.pdf.

Planeta y Paz, y Project Counselling Service -PCS. (2012). La cuestión Agraria en Colombia: Tierra, desarrollo y paz. Documento de trabajo.Memorias ciclo de conversatorios (documento de trabajo) [en línea]. Recuperado de http://www.hss.de/fileadmin/americalatina/ Colombia/downloads/cuestion_agraria.pdf

Programa de las Naciones Unidas para el Desarrollo -PNUD. (Agosto-septiembre de 201 I). ABC de la Ley de Víctimas y Restitución de Tierras.Hechos de Paz (edición especial), Año 7 (6I). Versión en línea disponible en http://www.pnud.org.co/hechosdepaz/echos/pdf/6I. pdf

Restrepo, J. C. (20I I). Política integral de tierras: Sector agropecuario locomotora de crecimiento. Cuadernos de Fedesarrollo: La política comercial del sector agrícola en Colombia (38), II-2I. http://www.repository.fedesarrollo.org.co/bitstream/I I445/I6I/I/CDF_No_38_ Mayo_20II.pdf.

Restrepo, J. C. (2013). Implementación de la política integral de tierras 2010-20I3. Acciones y resultados obtenidos en la materialización de las políticas de formalización, restitución administrativa y recuperación de baldíos. Bogotá, D. C.: Ministerio de Agricultura y Desarrollo Rural. Versión en línea recuperada dehttp://es.slideshare.net/YesidRiveral/informe-ministerio-de-agricultura

Restrepo, J. C. y Bernal, A. (20I4). La cuestión agraria: Tierra y postconflicto en Colombia. Bogotá, D. C.: Debate.

Rettberg, A. (2003). Diseñar el futuro: Una revisión de los dilemas de la construcción de paz para el postconflicto. Revista de Estudios Sociales (15), 15-28.

Rettberg, A. (2012). Construcción de paz en Colombia: Contexto y balance. En A. Rettberg (comp.) Construcción de paz en Colombia (pp. 3-50) Bogotá, D. C.: Ediciones Uniandes.

Reyes, A. (1978). Latifundio y poder político: La hacienda ganadera en Sucre. Bogotá, D. C.: Cinep.

Rodríguez, C. (Enero-junio de 20I4).La ley de restitución de tierras desde la perspectiva de género. Justicia Juris, I0(I), 53-65. Versión en línea disponible en http://www.scielo.org.co/pdf/jusju/vIOnl/vIOnla06.pdf

Sabogal, R., Machado, A., Éder, A. y Navarro, A. (2013). Dilemas del Postconflicto. En Facultad de Ciencias Sociales y Observatorio de Paz de la Universidad Jorge Tadeo Lozano (presidencia), Segundo panel llevado a cabo en el III Foro Colombiano en Construcción de Paz: “Justicia Transicional, Postconflicto y Construcción de Paz". Universidad Jorge Tadeo Lozano, Bogotá, D. C., Colombia. 
Sánchez, N. C. y Uprimny, R. (2010). Propuestas para una restitución de tierras transformadora. En C. Díaz (ed.), Tareas pendientes: Propuestas para la formulación de políticas públicas de reparación en Colombia (pp. 193-268). Versión en línea disponible en http://www. minjusticia.gov.co/Portals/0/Foros\%20Justicia\%20Transicional/TAREAS_PENDIENTES.pdf

Serrano, R. y Acevedo, M. (Enero-junio de 2013). Reflexiones en torno a la aplicación de la Ley 1448 de 201 I y la restitución de tierras en Colombia. Revista Facultad de Derecho y Ciencias Políticas, 43(I I8), 533-566. Versión en línea disponible en http://www.scielo.org.co/pdf/ rfdcp/v43nIl9/v43nl I9a03.pdf

Stake, R. (1998) Investigación con estudio de caso. Madrid: Morata.

Tadloui, S. (2012). Los retos cruzados de la reforma agraria, el desarrollo rural y la reconciliación: Análisis de un instrumento de política pública en el caso de Sandrana y Samaría. Opera, ( 12), 109-I 34. Versión en línea disponibleen http://www.redalyc.org/pdf/675/67530270006. pdf

Tobasura, I. (20 I I). De campesinos a empresarios: La retórica neoliberal de la política agraria en Colombia. Espacio abierto, 20(I4), 64-657. Versión en línea disponible en http://www.redalyc.org/articulo.oa?id=|2220531005

Ugarriza, J., Nuzzio, E., Orozco, M. M., Rodríguez, A., Martínez, U. y Gutiérrez, N. (2013). Experimentos sobre reconciliación política en Colombia [en línea]. Recuperado de http://www.urosario.edu.co/jurisprudencia/Experimentos-sobre-reconciliacion-politica-en-Colo/ ur/Postconflicto/

Universidad de San Buenaventura - Educación Continua. (2014). Diplomatura “Ley de víctimas y restitución de tierras": Ley I448 de 201 I (Programa y contenidos del diplomado) [en línea]. Recuperado de http://www.usbmed.edu.co/index.php/programas/educacion-continua/2uncategorised/452-ley-victimas-restitucion-tierras.

Varona, J. (Abril de 20I4). Posconflicto y paz: Un camino incierto (editorial). Revista Cipol, (4), 2-3. 